MATEC Web of Conferences 33, 03022 (2015)

DOI: $10.1051 /$ matecconf/ 20153303022

(C) Owned by the authors, published by EDP Sciences, 2015

\title{
Optimization of Automated Crystal Orientation and Phase Mapping in TEM Applied to Ni-Ti All Round Shape Memory Alloy
}

\author{
Xiayang $\mathrm{Yao}^{1}$, Yuanyuan $\mathrm{Li}^{2}$, Shanshan $\mathrm{Cao}^{2}$, Xiao $\mathrm{Ma}^{2}$, Xin-ping Zhang ${ }^{2}$ and Dominique Schryvers ${ }^{1}$ \\ ${ }^{1} E M A T$, University of Antwerp, Groenenborgerlaan 171, B-2020 Antwerp, Belgium; \\ ${ }^{2}$ School of Materials Science and Engineering, South China University of Technology, Guangzhou 510640, China
}

\begin{abstract}
A new application which focuses on an artificial sphincter fabricated by Ni-Ti SMAs for human implantation is under investigation by applying the all-round shape memory effect with precise control of the phase transformation temperatures. In this study, a Ni51at.\%-Ti alloy was fabricated by arc melting with fast solidification, followed by a proper strained aging which induces the two way shape memory effect needed for this particular application. Differential scanning calorimetry was used to investigate the thermal behavior and transmission electron microscopy was used for studying the microstructure of the alloys. With the latter the novel technique of automated crystal orientation microscopy is used and optimized to obtain phase and orientation mapping of the various structures.
\end{abstract}

\section{Introduction}

The attempts for finding new applications of Ni-Ti alloys are still continuing even after half a century's investigations of this material. In the present work, we investigated a new application which focuses on an artificial sphincter fabricated by Ni-Ti SMAs for human implantation. Such a sphincter assists the opening and closing of veins, bowels, etc. as shown in figure 1a and 1b. A Ni-Ti artificial sphincter will have several advantages over existing ones, such as good corrosion resistance and easier controllability [1]. The design is based on the all-round shape memory effect (ARSME) of $\mathrm{Ni}-\mathrm{Ti}$ which was first reported by Nishida and Honma [2]. The ARSME is actually a special and remarkable case of the well-known two way shape memory effect (TWSME) and which describes the effect that the alloy remembers one shape in its parent phase and when temperature decreases to below the phase transformation temperature, the shape will dramatically change to the opposite side as shown in figure 1c. This process can always be repeated in following heating and cooling processes after proper treatment of the alloy. As with conventional TWSME, the mechanism of this phenomenon is mainly controlled by the existence of $\mathrm{Ni}_{4} \mathrm{Ti}_{3}$ precipitates inside the alloy.[3]

$\mathrm{Ni}_{4} \mathrm{Ti}_{3}$ is an intermediate phase in the Ni-Ti alloy which appears as precipitates with a lenticular shape and central plane parallel to the crystallographic $\{111\}_{\mathrm{B} 2}$ planes in the B2 matrix.[4] There are in total eight possible $\mathrm{Ni}_{4} \mathrm{Ti}_{3}$ variants, but since two-by-two of these variants share the

\footnotetext{
a Corresponding author: nick.schryvers@uantwerpen.be
}

same habit plane, only four different orientation variants are typically considered [5]. Due to the orientation relationship with the matrix, usually maximum three variants are recognised in one single transmission electron microscopy image. These coherent precipitates could control the phase transformation because there exist strain fields around precipitates [6-8] as well as depletion of $\mathrm{Ni}$ in the surrounding matrix $[8,9]$.

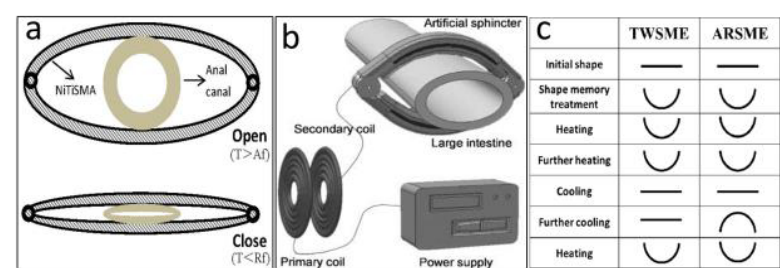

Figure 1 An illustration of, (a) and (b) application of Ni-Ti as artificial sphincter; (c) all round shape memory effect in comparison with the conventional two way effect.

The effect of $\mathrm{Ni}_{4} \mathrm{Ti}_{3}$ precipitates on the ARSME is mainly due to the particular growth and orientation of the precipitates during the training process. Due to the bending different strains occur in different regions $[10$, 11]: a tensile stress on the upper side and a compression stress on the lower side. The difference in strain distribution directly causes a difference in preferential orientation of the $\mathrm{Ni}_{4} \mathrm{Ti}_{3}$ precipitates and these differences will guide the ensuing phase transformation. A quantitative study of such differences in this all-round shape memory alloy will help to improve the phase transformation performance. 


\section{Experimental}

The samples were prepared from electrolytic nickel (purity $\geq 99.9 \%$ ) and titanium sponge (purity $\geq 99.7 \%$ ) by arc melting. The nominal composition of the alloy is Ni51at\%-Ti and the sample was formed by fast cool casting after arc melting. The size of the alloy stripe after casting is $70 \times 8 \times 0.5 \mathrm{~mm}$. A solid solution treatment was applied to homogenize the composition at $850^{\circ} \mathrm{C}$ for $3 \mathrm{~h}$ with Ar protection and followed by water quench. After solid solution, the alloys were divided into two groups. Those in the first group were placed in an arch shaped die with different aging parameters: $250^{\circ} \mathrm{C}, 300^{\circ} \mathrm{C}, 350^{\circ} \mathrm{C}$ and $400^{\circ} \mathrm{C}$, for $1 \mathrm{~h}, 10 \mathrm{~h}$ and $100 \mathrm{~h}$ each. The second group went through the same aging time and temperature but without the use of the arch die, which means that the alloys in the second group were aged under stress free conditions.

Differential Scanning Calorimetry (DSC) was applied to investigate the phase transformation properties and Transmission Electron Microscopy (TEM) was used to analyse the microstructure of the alloy. Automated crystal orientation mapping (ACOM-TEM) was used to further quantify the microstructure of the alloy. This device can acquire similar results as an Electron Backscatter Diffraction (EBSD) device but at a much smaller scale. In the present set-up in a Tecnai G2 microscope equipped with FEG, a resolution of $3 \mathrm{~nm}$ could be obtained. In the ACOM-TEM instrument from NanoMEGAS [12, 13] electron diffraction spot patterns (contrary to Kikuchi line patterns in an EBSD) are collected with an external CCD camera, capturing the diffraction pattern on the small phosphorous screen inside the column, while the sample area of interest is scanned by the electron beam. Local crystallographic orientation and/or phase are identified through an algorithm that compares the recorded electron diffraction spot patterns with pre- simulated kinematical templates for all possible orientations and/or phases of a number of pre-selected structures.[14] From the data acquired by ACOM-TEM, orientation as well as phase maps can be obtained together with quantitative information such as including size, distribution, volume fraction etc. of precipitates.

The DSC curve in figure $7 \mathrm{c}$ indicates that the samples contain $\mathrm{R}$ phase at room temperature. In order to allow for a clear distinction between B2 matrix and precipitates without the interference of $\mathrm{R}$ phase, in-situ heating was applied during the entire collection process of orientation mapping at $200{ }^{\circ} \mathrm{C}$, thus all the acquired data are with the matrix in the austenite state.

\section{Results and discussion}

As mentioned above, the application of ACOM-TEM is based on obtaining and identifying a series of diffraction spot patterns of a set area of the sample, so the whole process includes two parts: diffraction pattern acquisition and calculation. Thus, the reliability of the final results is based on the combination of both an experimental and a simulation part. For most cases, the default settings of the instrument are sufficient to obtain proper orientation and phase mapping results. Unfortunately, however, the present $\mathrm{Ni}-\mathrm{Ti}$ alloys proved to be an unusual case, for several reasons.

The areas of investigation contain both Ni-Ti B2 matrix and $\mathrm{Ni}_{4} \mathrm{Ti}_{3}$ precipitates. The reciprocal space of the $\mathrm{B} 2$ matrix is that of the common BCC structure but without systematic extinctions due to the ordering of the two kinds of atoms. The reciprocal space of $\mathrm{Ni}_{4} \mathrm{Ti}_{3}$ precipitates shares several basic reflections with that of the Ni-Ti B2 matrix, but rows of superspots appear in between the basic reflections. In other words, in kinematical conditions these superspots are the only sign to distinguish between the B2 matrix and the precipitates and for nanoscale precipitates, these superspots never have very high intensity. [15]

In order to allow the best matching between the experimental patterns and the simulated kinematical patterns, the system allows the use of electron beam precession during pattern acquisition. The main advantage of precession is to remove strong dynamical effects from the patterns leaving near-kinematical data, eliminating, e.g., extra spots due to double diffraction. Also, when using precession small specimen-tilt changes are averaged out and the intensity of the symmetric spots increases. Figures 2 and 3 show a comparison between ACOM-TEM results with and without precession, respectively, of a stress-free aged sample.

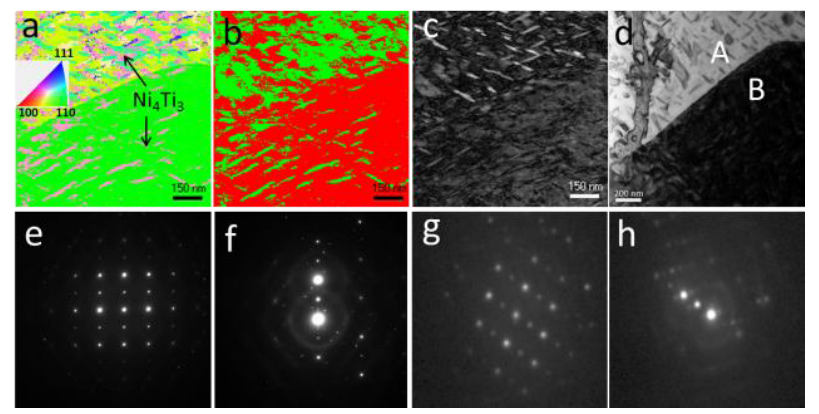

Figure 2 ACOM-TEM and conventional TEM results of a stress free aged sample $\left(400^{\circ} \mathrm{C}, 100 \mathrm{~h}\right)$ without precession, (a) orientation mapping (triangle corresponds with the B2 lattice), (b) phase mapping (red for B2 matrix and green for $\mathrm{Ni}_{4} \mathrm{Ti}_{3}$ precipitates), (c) reliability mapping, (d) conventional bright field image of the same region, (e) and (f) selected area diffraction patterns, e from grain $\mathrm{B}$ and $\mathrm{f}$ from grain $\mathrm{A}$ in $\mathrm{d},(\mathrm{g})$ and (h) uncorrected diffraction patterns acquired by ACOMTEM, $g$ from grain $B$ and $h$ from grain $A$.

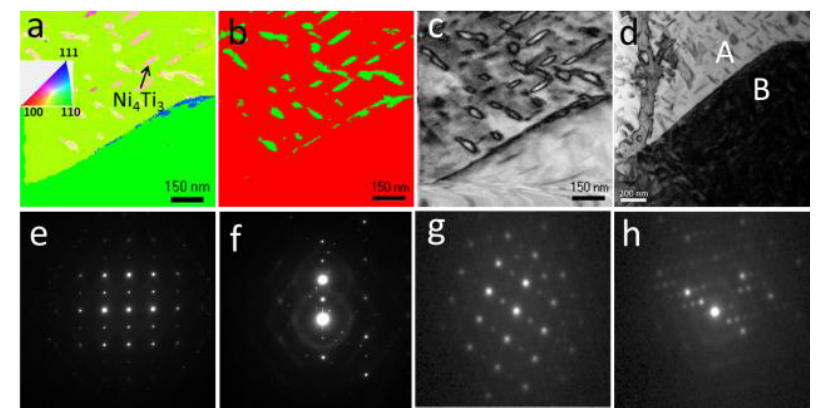

Figure 3 ACOM-TEM and conventional TEM results of the same sample and area of figure 2, now obtained with precession, (a) orientation mapping, (b) phase mapping (red for B2 matrix and green for $\mathrm{Ni}_{4} \mathrm{Ti}_{3}$ precipitates), (c) reliability 
mapping, (d) conventional bright field image of the same region, (e) and (f) selected area diffraction patterns, e from grain $\mathrm{B}$ and $\mathrm{f}$ from grain $\mathrm{A}$ in $\mathrm{d},(\mathrm{g})$ and $(\mathrm{h})$ uncorrected diffraction patterns acquired by ACOM-TEM, $g$ from grain $\mathrm{B}$ and $\mathrm{h}$ from grain A.

Figures $2 \mathrm{a}$ and $2 \mathrm{~b}$ are ACOM-TEM images acquired without precession while figures $3 \mathrm{a}$ and $3 \mathrm{~b}$ are corresponding images obtained with precession, the latter with an opening angle of $0.8^{\circ}$. Figures $2 \mathrm{c}$ and $3 \mathrm{c}$ are reliability maps of the corresponding orientation and phase calculation. The reliability describes how well acquired data is matched with possible templates. For each pixel on the map, a higher reliability means fewer templates match properly with the corresponding diffraction pattern, in other words, this means a higher accuracy for the matching results. On reliability maps, this judgement is indicated by the grey scale gradation. A higher reliability shows a brighter colour while a lower reliability is shown darker.

In the observed area, two grains indicated as grain A and $B$ in figure $2 d$ are distinguished: the orientation of grain $\mathrm{B}$ is very close to the $[110]_{\mathrm{B} 2}$ zone axis while grain $\mathrm{A}$ is off zone axis with only one $001_{\mathrm{B} 2}$ row of reflections being visible, as can be seen from the conventional selected area diffraction patterns shown in (e) and (f), respectively. In both figures 2 and 3, the patterns in (g) and $(\mathrm{h})$ are obtained at a selected scan position within the area shown in (d) and by the ACOM-TEM attachment, which yields distorted patterns due to the use of the small phosphorous screen. This distortion is corrected by calibration of the software before the template matching and will not be further discussed here. Comparing figures $2 \mathrm{~h}$ and $3 \mathrm{~h}$, which are from the ACOM-TEM results of grain $\mathrm{A}$, it is clear that when using precession, the number of superspots from the precipitates increases, which will improve reliability since the template matching will be more accurate. In fact, the intensity of all reflections changes when precession is used, but this is easier seen when comparing figures $2 \mathrm{~g}$ and $3 \mathrm{~g}$ (although the effect is also visible in the B2 reflections of figures $2 \mathrm{~h}$ and 3h). Thus, from the view of diffraction pattern's quality, precession enhances both the intensity and the number of spots, especially weak superspots.

When comparing figures $2 \mathrm{~b}$ and $3 \mathrm{~b}$, one notices a large difference in the number of identified $\mathrm{Ni}_{4} \mathrm{Ti}_{3}$ precipitates with much fewer precipitates being recognized when precession is used. This may at first be a surprise, since the superspots are better seen in precession mode and one would thus expect a better match with the $\mathrm{Ni}_{4} \mathrm{Ti}_{3}$ structure. The main reason for the loss in recognition of precipitates in the precession mode is due to a lowered lateral resolution as a result of the precessing beam which yields an enlarged area from which a single pattern (i.e., the superposition of all obtained patterns with precession at a given scan position) is obtained. As a result, also the average size of precipitates in $2 \mathrm{a} / \mathrm{b}$ is smaller than in $3 \mathrm{a} / \mathrm{b}$ since this effect is especially critical for the smaller precipitates.

Although more precipitates are observed without precession, the overall reliability is lower due to the dynamical effects not taken into account by the simulating software. As for the observed $\mathrm{Ni}_{4} \mathrm{Ti}_{3}$ precipitates, the reliability is good in grain A in both cases, but for the matrix part, reliability greatly increases in the map with precession.

To conclude from the above, precession enhances the number of detected diffraction spots. It decreases the lateral resolution and increases reliability of matrix while it has little effect on the reliability of the observed precipitates.

However, we still need to understand why the ACOMTEM technique finds more precipitates in grain A than in grain B. Since both grains have received the same thermal treatment and are next to one another in the sample, in this case indicating a similar foil thickness, the only difference can be the orientation condition in the microscope. The effects of precession can enhance this difference. It is clear that the precipitates are better recognized in the grain which is relatively far away from a zone axis, such as grain $\mathrm{A}$, which can be explained by the fact that, at least for $\mathrm{Ni}_{4} \mathrm{Ti}_{3}$, more intensity is scattered into superspots than for a grain such as B, which is very close to a simple crystallographic zone, here $[110]_{\mathrm{B} 2}$, only showing weak superspots. In both cases, the precession improves the quality of the template matching and thus reduces the noise in the mapping but apparently this effect primarily plays on the best recognizable features, i.e., the precipitates in grain A and the matrix in grain B. In grain $\mathrm{A}$ this improves the overall image quality and the matrix/precipitates interfaces become clearer (although some small precipitates will have been lost), while in grain B the precipitate information is completely lost when using precession. One should thus carefully choose the imaging conditions for each grain and for the $\mathrm{Ni}_{4} \mathrm{Ti}_{3}$ precipitates an off zone orientation showing many superspots that can be enhanced by precession seems to be the best option.

However, in practice there is another parameter which is related with both precession and tilting conditions while it also has a strong effect on the final calculated results. This parameter is the camera length (CL). In principle, for a well-calibrated TEM instrument, the value of CL for in-column detectors is known. For the external ACOMTEM detector, the value directly obtained from TEM is not reliable and the ACOM software calculates the correct CL, based on one of the acquired known diffraction patterns selected by the operator. In practice, the CL calculation will be more accurate if the diffraction pattern is more close to a zone axis because this provides more spots with a homogeneous intensity for the software to detect and a reliable CL determination is a basic need for the quality of the orientation and phase mapping. This implies that the conditions optimized for the template matching, as mentioned in the previous paragraph, are not ideal for the calibration of the CL, which is further worsened for more complicated patterns such as the one with $\mathrm{Ni}_{4} \mathrm{Ti}_{3}$ superspots. Moreover, a slight mistake in the calibrated CL will result in a sizable change of the diffraction pattern template, and for the Ni-Ti case, such a change is difficult for the software to distinguish.

The best condition to collect data in the present sample was thus found to be: tilting grains close to a low index zone axis without the use of precession, i.e., the case of 
grain $B$ in figure $2 \mathrm{~b}$. This ensures a reliable $\mathrm{CL}$ calculation and a clear recognition of the boundary of precipitates.

Apart from the tilting condition, the CL calibration and the use of precession, there are still other parameters affecting orientation and phase mapping results, and which are related with the calculations of the diffraction patterns. The default setting of the template generator (and which was used in the above examples) works well for most cases, as seen from the example shown in figure 4 in which $\mathrm{NiTi}_{2}$ precipitates are distributed at grain boundaries in a Ni-Ti B2 sample.

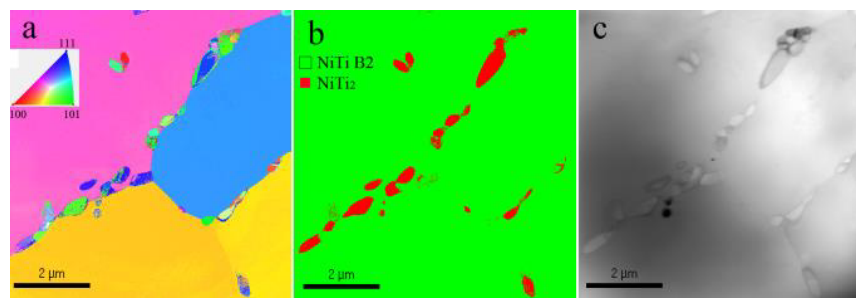

Figure 4 ACOM-TEM and conventional TEM image of Ni-Ti sample with $\mathrm{NiTi}_{2}$ precipitates at grain boundaries, (a) orientation mapping, (b) phase mapping, (c) conventional TEM bright field image.

Both structures are well recognized and separated by the system, even when using the default settings. This is because $\mathrm{Ni}$-Ti $\mathrm{B} 2$ and $\mathrm{NiTi}_{2}$ have very different lattice structures and thus the corresponding diffraction patterns can well be distinguished. As seen above, for the case of $\mathrm{Ni}_{4} \mathrm{Ti}_{3}$ precipitates in a Ni-Ti B2 matrix, these conditions not necessarily yield sharp maps. In practice it is found that the choice for the value of the excitation error also plays an important role. The excitation error describes that when the Bragg condition is not fully respected for a given reflection, the spot can still appear but with a lower intensity. For the template generation, such a decrease of the intensity is obtained by reducing the intensity linearly with the deviation from the Bragg angle, with the excitation error as a tunable parameter. Figures 5 and 6 give example of comparison of the influence of the excitation error.
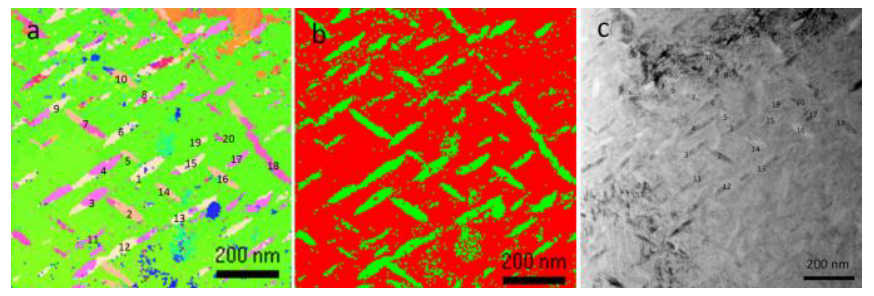

Figure 5 ACOM-TEM and conventional TEM results of a stress free aged Ni-Ti sample using the default excitation error of 1.0, (a) orientation mapping, (b) phase mapping (red for B2 matrix and green for $\mathrm{Ni}_{4} \mathrm{Ti}_{3}$ precipitates), (c) conventional TEM bright field image.

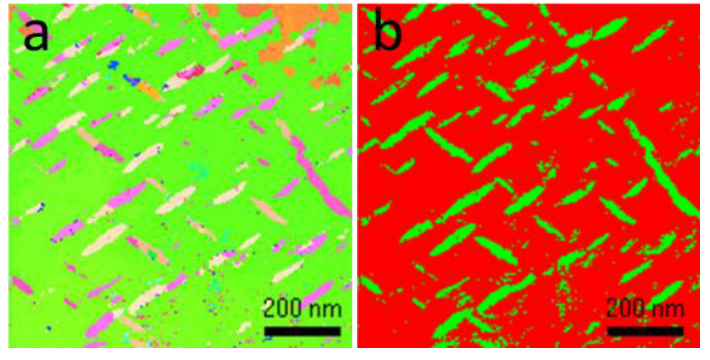

Figure 6 ACOM-TEM results of the same area as in figure 5, now using a small excitation error of 0.25 , (a) orientation mapping, (b) phase mapping (red for B2 matrix and green for $\mathrm{Ni}_{4} \mathrm{Ti}_{3}$ precipitates).

Figure 5 is obtained from a calculation using the default value for the excitation error (1.0) for the template generation of the $\mathrm{Ni}_{4} \mathrm{Ti}_{3}$ patterns, while figure 6 is obtained using a small excitation error $(0.25)$. A visual inspection immediately shows that the case of figure 5 contains more erratic pixels than that of figure 6, so lowering the excitation error might further improve the quality of the results. Here it should be mentioned that the present data set also reveals the two ordering variants for each of the orientation variants, as seen by the double colouring in some precipitates.

Figure 7 gives one application data of the ACOM-TEM technique on the ARSME sample, obtained from a sample taken from the bottom area of the arc shaped unit and treated for $100 \mathrm{~h}$ at $400^{\circ} \mathrm{C}$ under constant stress. The results clearly show the preferential appearance of $\mathrm{Ni}_{4} \mathrm{Ti}_{3}$ precipitates, orientation map figure $7 \mathrm{a}$ shows only one variant of precipitates, again with both ordering variants inside and $7 \mathrm{~b}$ clearly distinguishes matrix and precipitates in different colour. After the noise spots are eliminated by an automated MATLAB cleaning process, the precipitate volume density in figure $7 \mathrm{~b}$ is calculated to be $21 \%$.

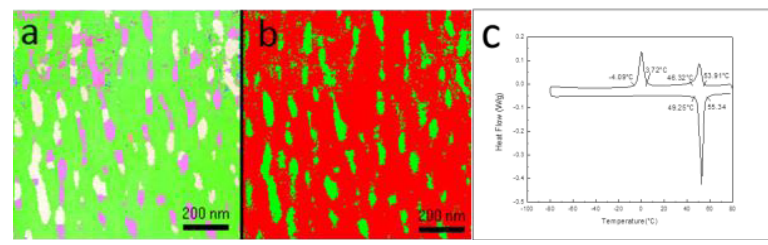

Figure 7 ACOM-TEM results of stress assisted aged Ni-Ti sample $\left(400^{\circ} \mathrm{C}, 100 \mathrm{~h}\right)$, (a) orientation mapping, (b) phase mapping (red for $\mathrm{B} 2$ matrix and green for $\mathrm{Ni}_{4} \mathrm{Ti}_{3}$ precipitates), (c) DSC curve

\section{Conclusions}

ACOM-TEM as a new technique to obtain quantified orientation and phase information with a higher resolution than conventional EBSD mapping was used to study the density of nanoscale $\mathrm{Ni}_{4} \mathrm{Ti}_{3}$ precipitates in a $\mathrm{Ni}$ $\mathrm{Ti}$ all-round shape memory alloy. In order to properly determine the precipitates, the technique was optimized to get reliable results on the nanoscaled precipitates. It is concluded that the tilting condition is an important parameter for obtaining good results; the grain should not be too far away from a low index zone axis but also 
should not be observed perfectly in zone. In this condition the primary beam precession method should not be used since it lowers the lateral resolution. However, if this tilting condition cannot be satisfied, precession could improve collected data by increasing the intensity in superspots. Also, an excitation error smaller than the default value should be set for the template generation of $\mathrm{Ni}_{4} \mathrm{Ti}_{3}$ precipitates.

\section{Acknowledgement}

X. Yao gratefully acknowledges the Chinese Scholarship Council (CSC) for providing a PhD scholarship.

\section{References}

1. H. Liu, Y. Luo, M. Higa, X. Zhang, Y. Saijo, Y. Shiraishi, K. Sekine and T. Yambe. J Artif Organs. 10, 223-7,(2007)

2. M. Nishida and T. Honma. Scripta Metallurgica. 18, 1293-1298,(1984)

3. R. Kainuma, M. Matsumoto and T. Honma. Proceedings of the International Conference on Martensitic Transformations. ICOMAT-86. 717722,(1986)

4. W. Tirry, D. Schryvers, K. Jorissen and D. Lamoen. Acta Crystallogr B. 62, 966-71,(2006)

5. M. Nishida and C.M. Wayman. Materials Science and Engineering. 93, 191-203,(1987)

6. W. Tirry and D. Schryvers. Nat Mater. 8, 7527,(2009)

7. W. Tirry and D. Schryvers. Acta Materialia. 53, 1041-1049,(2005)

8. N. Zhou, C. Shen, M.F.X. Wagner, G. Eggeler, M.J. Mills and Y. Wang. Acta Materialia. 58, 66856694,(2010)

9. Z. Yang and D. Schryvers. Micron. 37, 503507,(2006)

10. M.R. Doddamani and S.M. Kulkarni. Finite Element Analysis - Applications in Mechanical Engineering. 2012)

11. J. Khalil-Allafi, A. Dlouhy and G. Eggeler. Acta Materialia. 50, 4255-4274,(2002)

12. E.F. Rauch and L. Dupuy. Archives of Metallurgy and Materials. Vol. 50, iss. 1, 87-99,(2005)

13. E.F. Rauch and M. Veron. Materialwissenschaft und Werkstofftechnik. 36, 552-556,(2005)

14. E.F. Rauch and M. Véron. Materials Characterization. 98, 1-9,(2014)

15. T. Tadaki, Y. Nakata, K. Shimizu, Rsquo, Ichi and K. Otsuka. Transactions of the Japan Institute of Metals. 27, 731-740,(1986) 
